\section{Künstliche Intelligenz schlägt Hautärzte bei Melanom-Diagnose}

Brinker T] et al. Deep Learning Outperformed 136 of 157 Dermatologists in a Head-to-Head Dermoscopic Melanoma Image Classification Task. Eur J Cancer 2019; 113: 47 - 54 https://doi.org/10.1016/j.ejca.2019.04.001

Wissenschaftler des Deutschen Krebsforschungszentrums (DKFZ), der Universitäts-Hautklinik und des Nationalen Centrums für Tumorerkrankungen (NCT) Heidelberg haben einen Algorithmus programmiert, der Hautveränderungen digital beurteilen kann. In einer Studie traten 157 Hautärzte von zwölf deutschen Universitätskliniken gegen die künstliche Intelligenz an: Sowohl Ärzte als auch Algorithmus beurteilten Bilder danach, ob es sich um ein Muttermal oder ein Melanom handelt. Am Ende war die künstliche Intelligenz präziser.

Für 100 Bilder von Hautauffälligkeiten, davon 20 gesichert schwarzer Hautkrebs und 80 gutartige Muttermale, mussten die Dermatologen das weitere Vorgehen bestimmen: entweder eine Biopsie durchführen oder dem Patienten von der Gewebeprobe abraten. Dieselben 100 Bilder wurden anschließend von einem zuvor mit 12378 anderen Bildern trainierten Algorithmus automatisiert bewertet. Nur 7 der 157 Dermatologen schnitten besser als der Algorithmus ab, 14 erzielten gleich gute Ergebnisse und 136 hatten schlechtere Ergebnisse. Im Durchschnitt war der Algorithmus präziser in der Beurteilung der Hauttumoren als die Hautärzte. Dabei spielte es keine Rolle, welche Position und Erfahrung der Arzt hatte: Im Durchschnitt waren Assistenzärzte über Fach- und Oberärzte bis zum Chefarzt dem Algorithmus unterlegen.

Eine Diagnose von Hautveränderungen allein durch den Algorithmus empfehlen die Forscher trotzdem nicht. Ein Einsatz auf mobilen Endgeräten ist zwar in bestimmten Situationen denkbar, setzt die Patienten aber derzeit noch zu hohen Risiken aus. Denn der Algorithmus kennt bisher nur zwei Diagnosen: Muttermal oder schwarzen Hautkrebs. Bei dieser Fragestellung ist die künstliche Intelligenz bei Bilddaten überlegen. „Die klinische Realität ist allerdings eine völlig andere: Ein Facharzt muss bei der körperlichen Untersuchung zwischen mehr als hundert Differenzialdiagnosen unterscheiden können, davon sind viele sehr selten, einige sind kaum allein am Bild zu erkennen, sondern brauchen weitere Informationen wie zum Beispiel Tasteindrücke“, berichtet Alexander Enk, Direktor der Universitäts-Hautklinik Heidelberg. Nach Meinung der Heidelberger Mediziner wird die künstliche Intelligenz den Arzt daher in naher Zukunft auch nicht ersetzen. Sie könnte die klinische Beurteilung von Hauttumoren jedoch sinnvoll ergänzen: „Es ist ähnlich wie beim Autopiloten im Flugzeug: Bei gutem Flugwetter und häufigen Strecken ist das Assistenzsystem hilfreich. Bei schwierigen Landungen muss ein erfahrener Pilot hingegen Verantwortung übernehmen. Das kann ein Computer so allein nicht leisten“, sagt Titus Brinker, Leiter der Studie und Wissenschaftler am DKFZ, NCT Heidelberg sowie Assistenzarzt an der Universitäts-Hautklinik Heidelberg.

Nach einer Pressemitteilung des Nationalen Centrums für Tumorerkrankungen (NCT) Heidelberg 